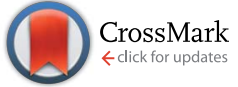

Cite this: RSC Adv., 2017, 7, 4000

\title{
Effect of different solvent systems on PHBV/PEO electrospun fibers
}

\author{
Yongjing $\mathrm{Xu}$, Liming Zou, * Hongwei Lu and Tingjie Kang
}

The selection of non-hazardous solvent systems is an important factor that can significantly influence fiber formation during polymer electrospinning. In this paper, poly(3-hydroxybutyrate-co-3-hydroxyvalerate) (PHBV)/polyethylene oxide (PEO) has been electrospun using different solvent systems to investigate the influence of different solution properties on nanofiber morphology and diameter, the thermal and mechanical properties, as well as the degradation kinetics of the electrospun fibers. The morphology, thermal and mechanical properties of PHBV/PEO electrospun fibers were characterized using scanning electron microscopy (SEM), thermogravimetric analysis (TG) and differential scanning calorimetry (DSC), and a universal testing machine, respectively. The results showed that the binary-solvent system (dichloromethane/ethanol DCM/EtOH) gave the finest defect-free fibers, and exhibited the best thermal and mechanical properties of all the single solvents (chloroform ( $\mathrm{CHL}$ ), dichloromethane (DCM)). Therefore, the effect of DCM/EtOH in different ratios on PHBV/PEO electrospun fibers was studied in detail. In brief, the DCM/EtOH solvent system was considered to be the best candidate for PHBV/PEO for electrospinning.

Received 14th November 2016 Accepted 29th December 2016

DOI: 10.1039/c6ra26783a

www.rsc.org/advances significantly affecting solution spinnability and the morphology of the electrospun fibers. Thus for successful electrospinning the selection of an appropriate solvent system is indispensable.

Luo et al. ${ }^{9}$ studied that the effect of 28 solvent systems on polymethylsilsesquioxane (PMSQ) nanofibers. Binary solvent systems were produced for electrospinning, while the solvent parameters were close to a good single solvent. PMSQ solutions of the same concentration in solvents of partial solubility showed better spinnability than solutions in solvents of high solubility. The binary solvent system mixing 2-nitropropane (high solubility) and dimethylsulphoxide (non-solvent), neither of which exhibited high volatility, highly porous electrospun fibres. It demonstrated that solvents with high solubility and volatility did not necessarily produce solutions good for electrospinning, and phase separation was induced by solubility difference in the electrospun polymer solution. Casasola et al. ${ }^{8}$ prepared poly lactic acid (PLA) in various pure solvents and binary-solvent systems, and investigated the effect of different solution properties on nanofibre morphology and diameter. It was found that, of all the solvent systems used, acetone/ dimethylformamide gave the highest fibre productivity and finest defect-free nanofibres. The results showed that the solvent properties, boiling point, viscosity, conductivity and surface tension, had a significant effect on process productivity, morphology and diameter distribution of the PLA nanofibres.

Poly(3-hydroxybutyrate-co-3-hydroxyvalerate) (PHBV) became attractive in the biomedical field due to its optical activity, biodegradability, biocompatibility and thermoplasticity. ${ }^{\mathbf{6}, 10}$ Furthermore, (R)-3-hydroxybutyric acid, the ultimate
State Key Laboratory for Modification of Chemical Fibers and Polymer Materials, College of Materials Science and Engineering, Donghua University, 2999 North People Road, Shanghai 201620, People's Republic of China. E-mail: lmzou@dhu. edu.cn 
degradation product of PHBV, is a constituent of blood. It was reported that PHBV could promote better cell proliferation, such as fibroblasts, keratinocytes and neural cells. ${ }^{\mathbf{1 1 - 1 3}}$ However, PHBV has been prevented its application in some biomedical fields by some shortcomings, such as poor mechanical performance, high crystallinity degree and inherent rigidity, and intrinsic hydrophobicity. ${ }^{\mathbf{1 4}}$ As the surface modification with hydrophilic polymers, polyethylene oxide (PEO) has been explored because of the outstanding properties, ${ }^{\mathbf{1 5}}$ including good solubility in water and in organic solvents, a lack of toxicity, no antigenicity, non-immunogenicity and hydrophilic, all of which are essential properties for tissue engineering.

Regarding the production of PHBV nanofibers, the effect of different solvents on fiber diameter has been previously reported. Meng et al. $^{\mathbf{1 6}}$ dissolved PHBV in hexafluoroisopropanol (HFIP), the fiber diameter were $600 \mathrm{~nm}$, and the cell culture experiments indicated that the PHBV nanofibrous scaffold accelerated the adhesion and growth of NIH3T3 cells, thus making the former a good scaffold for tissue engineering. Kuppan et al. ${ }^{\mathbf{1 1 , 1 4}}$ dissolved PHBV in a mixture of DCM and DMF $(9: 1)$ to form a $15 \%(w / v)$ solution. The average diameter of the PHBV fibers was $724 \mathrm{~nm}$. However the PHBV fibers were not uniform and smooth. Hyeong et $a .^{17}$ investigated a set of concentration to produce PHBV fibers. They dissolved PHBV in trifluoroethanol (TFE), the factors chosen were solvent composition, concentration, applied voltage and tip-to-collector distance. The $2 \mathrm{wt} \%$ PHBV-TFE solution appeared to be the best choice to get the finest diameter. Sombatmankhong et al. ${ }^{18}$ prepared PHBV fibers using chloroform as solvent. The average diameter of the as-spun fiber from PHBV solutions decreased with increasing collection distance and increased with increasing solution concentration and applied electrical potential. The average diameter of PHBV fibers ranged between 1.6 and $4 \mu \mathrm{m}$.

Considering the PHBV/PEO blend system, Bianco et al. dissolved PHBV/PEO blends of different composition in chloroform at a concentration of $20 \%$, and collected electrospun fibers with average diameter of 1.3-2.6 $\mu \mathrm{m}$. In another study, Xu et al. ${ }^{19}$ produced PHBV/PEO nanofibers with a diameter of 738$1098 \mathrm{~nm}$ from the DCM solution 10\%. However some beads were also collected in the PHBV/PEO 80/20 blend.

Based on the facts discussed above, the selection of an appropriate solvent or solvent system is essential for the production of homogeneous nanofibers in the electrospinning process. Generally, PHBV is dissolved in chloroform and fluorinated solvents. However a low toxicity solvent system would be preferable, which is beneficial to the environment.

The main objectives of this study were to produce $\mathrm{PHBV} / \mathrm{PEO}$ nanofibers using chloroform (CHL), dichloromethane (DCM) and DCM/EtOH as solvent, and to select a desirable solvent or solvent system as a carrier of PHBV/PEO for the optimization of electrospinning. It is important to underline that, to the best of our knowledge, the lower toxicity solvent system of DCM/EtOH has not been reported yet. Meanwhile, the addition of EtOH to the DCM solvent enable the production of nanofibers. In order to evaluate the influence of the solvent systems on the morphology, mean diameter, diameter distribution thermal and mechanical properties, in vitro degradation of $\mathrm{PHBV} / \mathrm{PEO}$ nanofibres, all nanofibers were characterized by means of scanning electron microscopy (SEM), Fourier transform infrared attenuated total reflectance spectroscopy (FTIR-ATR), thermal analysis (TG-DTG), differential scanning calorimetry (DSC), X-ray diffraction (XRD), while mechanical properties and in vitro degradation were determined by universal testing machine and in vitro degradation test, respectively. The most promising solvent system DCM/EtOH was studied in more detail and solution viscosity, surface tension and conductivity were measured.

\section{Materials and methods}

\subsection{Materials}

Poly(3-hydroxybutyrate-co-3-hydroxyvalerate) (PHBV) with 1.09 mol\% 3-hydroxyvalerate (HV) $\left(M_{\mathrm{w}}=2.67 \times 10^{5} \mathrm{~g} \mathrm{~mol}^{-1}\right)$, was purchased from Tianan Biologic Material CO., poly(ethylene oxide) (PEO, $M_{\mathrm{w}}=100000 \mathrm{~g} \mathrm{~mol}^{-1}$ ) powder was purchased from Aladdin, and chloroform (CHL) $\left(\mathrm{CHCl}_{3}, 99.5 \%\right)$, dichloromethane (DCM) $\left(\mathrm{CH}_{2} \mathrm{Cl}_{2}, 99.5 \%\right)$, and ethanol (EtOH, 99.7\%) were supplied by Shanghai Lingfeng Chemical Reagent CO. All reagents and solvents were used as purchased without any further purification.

\subsection{Preparation of $\mathrm{PHBV} / \mathrm{PEO}$ electrospun fibers}

The PHBV powder with PEO content (70/30 wt\%/wt\%) was dissolved in CHL, DCM and DCM/EtOH ( $6: 1 \mathrm{v} / \mathrm{v}$ ) respectively, and magnetically stirred for $12 \mathrm{~h}$ to obtain a $10 \% \mathrm{w} / \mathrm{v}$ polymer concentration. The effect of DCM/EtOH solvent system on fiber morphology was investigated: $\mathrm{PHBV} / \mathrm{PEO}$ was dissolved in variable proportion of DCM/EtOH $(9: 1,8: 1,7: 1,6: 1,5: 1$, $4: 1,3: 1)$ to obtain solutions $10 \% \mathrm{w} / \mathrm{v}$. The solution was filled in a $10 \mathrm{ml}$ syringe equipped with a $22 \mathrm{G}$ metallic needle, fixed in a digitally controlled syringe pump (KD scientific, MA, USA), and a rectangular steel plate covered with aluminum foil was used as collector. The parameters of PHBV/PEO electrospinning were applied according to the method developed by Xu et al., as previously reported. ${ }^{19}$

\subsection{Viscosity, conductivity, surface tension and vapor pressure of polymeric solutions}

The shear viscosity of polymeric solutions was measured using a rotating viscometer (Digital viscometer DV-79, Ni Run). The temperature of the solutions was kept at $25^{\circ} \mathrm{C}$ by a water jacket and a thermostatically controlled water bath. Every reading was recorded until reaching equilibrium. Electric conductivities were evaluated at $25{ }^{\circ} \mathrm{C}$ by means of a conductometer (DDS-307A, Lei $\mathrm{Ci}$, Shanghai). The surface tension measurements of all solutions were conducted with a surfactometer (JK99B, Zhong Chen, Shanghai). The vapor pressure of binary solution can be calculated according to Raoult's law, using the equation: ${ }^{20}$

$$
P=P_{\mathrm{a}} X_{\mathrm{a}}+P_{\mathrm{b}} X_{\mathrm{b}}
$$

where $P$ is equal to the sum of the vapor pressures of the binary solvent solution, $P_{\mathrm{a}}$ and $P_{\mathrm{b}}$ are the vapor pressure of the pure 
component, $X_{\mathrm{a}}$ and $X_{\mathrm{b}}$ are the mole fraction of the component in solution.

\subsection{Microstructural investigation of PHBV/PEO electrospun fibers}

Surface morphology. The surface morphology of all PHBV/ PEO electrospun fibers was analyzed using a scanning electron microscope (TM-1000, Japan) at an accelerating voltage of $10 \mathrm{kV}$. The samples were sputter coated with gold prior to imaging. The average fiber diameter and fiber diameter distributions were determined from considering about 100 different fibers by means of ImageJ software.

Porosity analysis. The porosity of $\mathrm{PHBV} / \mathrm{PEO}$ electrospun fibers was calculated using the following equations: ${ }^{21}$

$$
\text { Porosity }(\%)=\left(1-\frac{d}{D}\right) \times 100 \%
$$

where, $d$ and $D$ refer to the apparent density $\left(\mathrm{g} \mathrm{cm}^{-3}\right)$ of the electrospun fibers and the bulk density of polymer. Assuming the density of PHBV and PEO were unchanged during the fabrication process, and utilizing the actual densities of PHBV and PEO of $\rho_{\text {PHBV }}=1.23 \mathrm{~g} \mathrm{~cm}^{-3}$ and $\rho_{\text {PEO }}=1.125 \mathrm{~g} \mathrm{~cm}^{-3}$, respectively. The bulk densities $(D)$ and apparent density $(d)$ were calculated using the following equations: ${ }^{7}$

$$
\begin{array}{r}
D=\frac{M_{\mathrm{PHBV}}+M_{\mathrm{PEO}}}{\frac{M_{\mathrm{PHBV}}}{\rho_{\mathrm{PHBV}}}+\frac{M_{\mathrm{PEO}}}{\rho_{\mathrm{PEO}}}} \\
d=\frac{M_{\text {electrospun mats }}}{L \times W \times h}
\end{array}
$$

$M_{\mathrm{PHBV}}$ and $M_{\mathrm{PEO}}$ are the weight of PHBV and PEO in the composites; $M_{\text {electrospun mats }}$ is the weight of the samples, and $L$, $W$ and $h$ are the length, width and thickness of the samples. Five strips were chosen for each porosity calculation.

Fourier transform infrared spectroscopy. Infrared (IR) spectra of all mats were recorded on a Nicolet 8700 spectrophotometer, by ATR reflection method. In the measurement, 128 spectral scans were repeated over the wavenumber range of $650-4000 \mathrm{~cm}^{-1}$ with the resolution of $4 \mathrm{~cm}^{-1}$.

\subsection{Thermal properties analysis of PHBV/PEO electrospun fibers}

The thermal properties were tested by thermogravimetric analysis (TGA, Netzsch 209 F1) and differential scanning calorimetry (DSC, TA Instruments Q20). TG analysis was also conducted from 20 to $600{ }^{\circ} \mathrm{C}$ under nitrogen atmosphere at a heating rate of $10{ }^{\circ} \mathrm{C} \mathrm{min}^{-1}$. The actual weight ratio and thermal stability of electrospun mats can be determined from the weight loss curves.

In the DSC measurement, the melting temperature and crystallinity of the samples were investigated under nitrogen atmosphere. About 7-8 $\mathrm{mg}$ of the samples were first heated from $0-190{ }^{\circ} \mathrm{C}$ at a rate of $10{ }^{\circ} \mathrm{C} \mathrm{min}^{-1}$.

Melting temperatures $\left(T_{\mathrm{m}}\right)$ and melting enthalpy $\left(\Delta H_{\mathrm{m}}\right)$ were determined from the first heating scan. The degree of crystallinity $(\chi)$ was calculated from equation:

$$
\chi=\frac{\Delta H_{\mathrm{m}}}{f \cdot \Delta H_{\mathrm{m}}^{0}}
$$

where $\Delta H_{\mathrm{m}}^{0}$ represents the theoretical enthalpy value of fully crystalline polymers (i.e. $109 \mathrm{~J} \mathrm{~g}^{-1}$ and $196.8 \mathrm{~J} \mathrm{~g}^{-1}$ for $\mathrm{PHBV}^{22}$ and $\mathrm{PEO},{ }^{23}$ respectively); $\Delta H_{\mathrm{m}}$ indicates the melting enthalpy (J $\mathrm{g}^{-1}$ ) as calculated from DSC thermograms. $f$ is the weight fraction of PHBV or PEO in the hybrid mixture.

\subsection{Mechanical properties of PHBV/PEO electrospun fibers}

The mechanical properties of the PHBV/PEO electrospun fibers were evaluated using Universal Testing Machine (WDW3020, Changchun). All electrospun mats of $10 \mathrm{~mm} \times 80 \mathrm{~mm}(n=5)$ were used in this study. The ends of the samples were mounted on the gripping units of the tensile tester, and a load of $500 \mathrm{~N}$ at an extension rate of $5 \mathrm{~mm} \mathrm{~min}^{-1}$ was applied until failure.

\subsection{In vitro degradation of $\mathrm{PHBV} / \mathrm{PEO}$ electrospun fibers}

The PHBV/PEO electrospun fibers (dimensions: $2 \mathrm{~cm} \times 2 \mathrm{~cm}$; thickness $110 \mu \mathrm{m}$ ) were subjected to in vitro degradation by incubating the samples in phosphate buffered saline (PBS) solution at $37^{\circ} \mathrm{C}$ for 2 week and 4 week, and PBS was changed every alternate day. The samples were removed after respective time points and rinsed twice with distilled water. The samples were then dried in a vacuum oven at room temperatures for 1 week and the morphology change was recorded by using SEM. ${ }^{24}$ Further, the weight loss percentage of the samples was calculated using the following equation:

$$
\begin{aligned}
\text { Weight loss }(\%)= & \frac{\text { initial } M_{\mathrm{w}} \text { of sample }- \text { final } M_{\mathrm{w}} \text { of sample }}{\text { initial } M_{\mathrm{w}} \text { of sample }} \\
& \times 100 \%
\end{aligned}
$$

At least 3 samples were tested for each type of electrospun fibers and the results were expressed as mean \pm SD.

\section{Results and discussion}

\subsection{The effect of solvents on PHBV/PEO electrospun fiber morphology}

The morphologies of PHBV/PEO electrospun fibers produced with different solvents were observed with SEM, the results and fiber diameter distribution were shown in Fig. 1. As illustrated in Table 2, PHBV/PEO solutions of CHL, DCM and DCM/EtOH produced bead-free smooth electrospun fibers of average diameters $1983 \pm 365 \mathrm{~nm}, 857 \pm 424 \mathrm{~nm}$ and $783 \pm 161 \mathrm{~nm}$, respectively. Previous literature has shown that solvent properties, such as concentration, boiling point, dielectric constant, conductivity, surface tension, and viscosity, influence the electrospun fibre morphology. ${ }^{25}$ It is well known that the conductivity and dielectric constant have the similar effect on the fiber diameter. The solvent with higher conductivity and larger dielectric constant have a higher charge density in solution. Therefore, as the charges carried by the jet increased, the stronger elongation forces are imposed to the jets because of the 

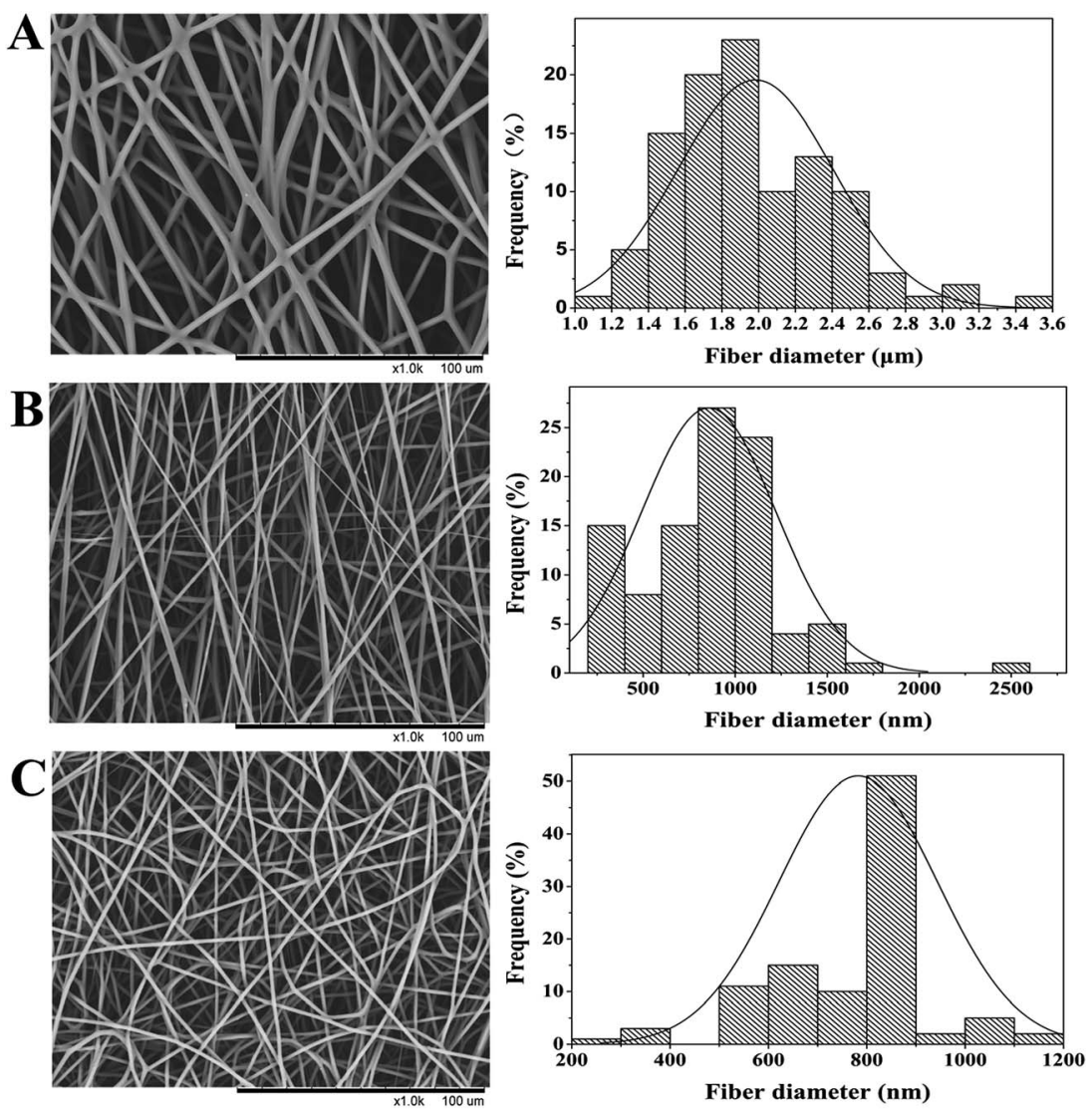

Fig. 1 SEM images of PHBV/PEO electrospun fibers with fiber diameter distribution obtained from 10\% (w/v) PHBV/PEO solution prepared by using different solvents: (A) CHL (B) DCM (C) DCM/EtOH.

Table 1 Properties of the solvents used in this study ( $\eta$ is the viscosity, $\varepsilon$ is the dielectric constant, $\sigma$ is the surface tension) (Smallwood I. Handbook of organic solvent properties, Halsted Press, London 1996)

\begin{tabular}{|c|c|c|c|c|c|c|}
\hline Solvent & $\begin{array}{l}\text { Boiling } \\
\text { point }\left({ }^{\circ} \mathrm{C}\right)\end{array}$ & $\begin{array}{l}\eta(\mathrm{mPa} \mathrm{s}) \\
\text { at } 20^{\circ} \mathrm{C}\end{array}$ & $\begin{array}{l}\text { Conductivity } \\
\left(\mu \mathrm{S} \mathrm{cm}^{-1}\right)\end{array}$ & $\varepsilon$ & $\begin{array}{l}\sigma\left(\mathrm{mN} \mathrm{m}^{-1}\right) \\
\text { at } 20{ }^{\circ} \mathrm{C}\end{array}$ & $\begin{array}{l}\text { Vapor pressure } \\
\text { at } 20^{\circ} \mathrm{C}(\mathrm{mmHg})\end{array}$ \\
\hline Chloroform - CHL & 61 & 0.563 & $1.0 \times 10^{-4}$ & 4.9 & 27.14 & 156 \\
\hline Ethanol - EtOH & 78 & 1.17 & $1.35 \times 10^{-13}$ & 25.7 & 22.27 & 45.7 \\
\hline
\end{tabular}

self-repulsion of the excess charges under the electrical field, leading to substantially straighter shape and smaller diameter of electrospun fibers. As the solution viscosity increased, the charged jet can not break up into small droplets but form the straight shape in electrospinning due to the increased chain entanglements (or the viscoelastic force). However, the increased viscoelastic force can prevent the jet segment from being stretched by the constant coulombic force, resulting in the larger fiber diameter. The solution in DCM has much higher conductivity and dielectric constant, and lower viscosity than that in $\mathrm{CHL}$, which leads to the thinner fiber diameter in DCM solvent. However, due to the high boiling point and vapor pressure of DCM (in Table 1), the Fig. 1B has revealed un-uniform and irregular electrospun fibers. Asran et al. ${ }^{20}$ and Qin et al. ${ }^{26}$ found the similar result on poly(caprolactone) (PCL) electrospun fibers. Therefore, of all solvents, DCM/EtOH was found to produce smooth and uniform fibers due to its relatively high conductivity and dielectric constant compared to the other single solvents, combined with relatively low surface tension.

As demonstrated in Table 2, the addition of EtOH in the DCM solvent enable the conductivity and boiling point increased, and the vapor pressure and surface tension decreased. For above reason, smooth defect-free electrospun fibers with a narrow diameter distribution were collected using the binary-solvent system. Hohman et al. ${ }^{27}$ reported that the dominant instability in electrospinning depended strongly on the static charge density of the jet, a high conductivity fluid or surface charge density can suppress the instability, and allowed the production of thinner and more uniform fibres with fewer defects. Solvent evaporation has been reported to decrease the elongation factor during electrospinning. ${ }^{28}$ Comparing with pure DCM, the DCM/EtOH with higher boiling point evaporate slower from the ejected charged jet, that can cause the viscoelastic properties of the jet to change, leading to a much lower diameter due to the stretching of the jet. 
Table 2 Conductivity, viscosity and surface tension of PHBV/PEO solutions and average fiber diameter and porosity of PHBV/PEO electrospun fiber

\begin{tabular}{|c|c|c|c|c|c|}
\hline Sample & $\begin{array}{l}\text { Conductivity } \\
\left(\mu \mathrm{S} \mathrm{cm}^{-1}\right)\end{array}$ & $\begin{array}{l}\text { Viscosity } \\
\text { (mPa s) }\end{array}$ & $\begin{array}{l}\text { Surface tension } \\
\left(\mathrm{mN} \mathrm{m}^{-1}\right)\end{array}$ & $\begin{array}{l}\text { Average fiber diameter } \\
(\mathrm{nm})\end{array}$ & $\begin{array}{l}\text { Porosity } \\
(\%)\end{array}$ \\
\hline CHL & $0.18 \pm 0.01$ & $96 \pm 2.3$ & $26.4 \pm 0.4$ & $1983 \pm 424$ & $80.4 \pm 1.45$ \\
\hline DCM/EtOH & $4.99 \pm 0.01$ & $76 \pm 2.0$ & $24.8 \pm 0.4$ & $783 \pm 161$ & $90.5 \pm 0.51$ \\
\hline EtOH & - & - & $20.1 \pm 0.7$ & - & - \\
\hline
\end{tabular}

As shown in Table 2, the binary-solvent system DCM/EtOH solution produced nanofibres with the thinnest diameter $(783 \mathrm{~nm})$, appear to agree with Lee et $a .^{29}$ Conductivity maybe the most important factor in the process of electrospinning. The high conductivity of solutions can result in the increase of surface charge of spinning jet, while stronger elongation forces are imposed to the jet, therefore, the defect-free, uniform nanofibers with a narrow diameter distribution were fabricated as shown in Fig. 1C.

Regarding the fiber diameter distribution, it was noticed that the homogenous electrospun fibers with thinner fiber diameter and narrow diameter distribution were prepared when the $\mathrm{PHBV} / \mathrm{PEO}$ dissolved in DCM/EtOH. This result can be attributed to the second solvent of EtOH with low vapor pressure ${ }^{\mathbf{2 0 , 3 0 , 3 1}}$ and surface tension, ${ }^{25,32}$ and high dielectric constant. In addition, the porosity of all electrospun fibers is $80-90 \%$, which is satisfied with the needs of tissue engineering.

\subsection{FTIR analysis of PHBV/PEO electrospun fiber}

The FTIR spectra of PHBV/PEO electrospun fiber with different solvents is presented in Fig. 2. As other researchers investigated, for all PHBV/PEO electrospun fibers, the $1722 \mathrm{~cm}^{-1}$ peak is assigned to the stretching band of the ester group $\mathrm{C}=\mathrm{O}$, which is sensitive to the crystalline phase; $2885 \mathrm{~cm}^{-1}, 2928 \mathrm{~cm}^{-1}$ and 2976 $\mathrm{cm}^{-1}$ peaks are corresponding to asymmetric and symmetric stretching of $\mathrm{C}-\mathrm{H}_{2} ; 1278 \mathrm{~cm}^{-1}$ and $1259 \mathrm{~cm}^{-1}$ peaks are assigned to asymmetric and symmetric stretching of $\mathrm{C}-\mathrm{O}-\mathrm{C} ; 1131 \mathrm{~cm}^{-1}$

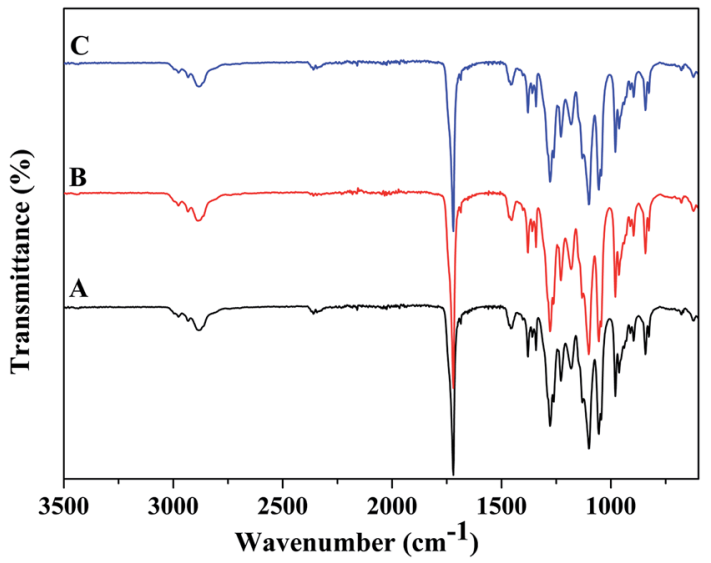

Fig. 2 FTIR spectra of PHBV/PEO electrospun fiber in different solvent: (A) CHL, (B) DCM, (C) DCM/EtOH. and $1101 \mathrm{~cm}^{-1}$ peaks are due to the asymmetric and symmetric stretching of $\mathrm{C}-\mathrm{O}$; the peak $1228 \mathrm{~cm}^{-1}$ is assigned to $\mathrm{CH}_{3}$ vibration, and $1183 \mathrm{~cm}^{-1}$ is also caused by C-O-C stretching; 1055 $\mathrm{cm}^{-1}$ peak is thought to be $\mathrm{C}-\mathrm{O}$ stretching and $\mathrm{CH}_{2}$ rocking. ${ }^{7,33}$ Whenever for any of the FTIR spectra of PHBV/PEO electrospun fiber in different solvent, there were no difference among them.

\subsection{DSC analysis of PHBV/PEO electrospun fiber}

Fig. 3 shows the DSC thermograms of PHBV/PEO electrospun fibers in different solvents, and the numerical data are summarized in Table 3. Fig. $3 \mathrm{a}$ and $\mathrm{b}$ represent the curves of the melting behavior of the first cycle and the second cycle showing samples without thermal history, while $3 \mathrm{c}$ reflects the crystallization behavior of samples.

It was noticed that the two distinct melting peaks appear in the Fig. 3a, implying that PHBV and PEO were also immiscible in the mixture, no matter whether the solvent was. In Table 3, the melting temperature $T_{\mathrm{m} 1}$ exhibited different values when $\mathrm{PHBV} / \mathrm{PEO}$ was dissolved in different solvent, i.e., $64.6{ }^{\circ} \mathrm{C}$ and 174.6 ${ }^{\circ} \mathrm{C}$ (in $\mathrm{CHL}$ solvent), $63.7{ }^{\circ} \mathrm{C}$ and $173.8{ }^{\circ} \mathrm{C}$ (in DCM solvent), $67.5^{\circ} \mathrm{C}$ and $176.2^{\circ} \mathrm{C}$ (in DCM/EtOH solvent). It can be found that when higher boiling point of solvent used, higher $T_{\mathrm{m}}$ of fibers was obtained. Like similarly the melting temperature $T_{\mathrm{m} 2}$ and crystallinity in different solvents have shown the same trend. These results are in close agreement with Chu's work. ${ }^{34}$ During electrospinning, when the polymer solution jet ejects from the needle tip and polymer chains are stretched in the direction of the electrostatic field (along the fiber direction), the surface area of the jet is dramatically increased during this process and this leads to an increased rate of solvent evaporation, the evaporative cooling during the loss of solvent leads to thermodynamic instability. Due to high boiling point or low vapor pressure, the rate of jet solidification was decreased and thereby the thermodynamic stability of $\mathrm{PHBV} / \mathrm{PEO}$ in DCM/EtOH solvent was better than that in CHL and DCM solvent.

The previous results indicate that the choice of the solvent with a suitable boiling point or vapor pressure is an important issue which will affect the crystallinity of the electrospun nanofibers, the solvent with a slower evaporation rate, give more time for the crystals rearrangement and facilitate the development of a highly crystalline structure. ${ }^{35}$ As a result, the crystallinity of PHBV/PEO electrospun fiber in DCM/EtOH solvent was higher compared with CHL and DCM solvent.

As illustrated in Table 3, the crystallization temperature $T_{\mathrm{C}}$ of $\mathrm{PHBV} / \mathrm{PEO}$ electrospun fiber in the binary-solvent was higher 

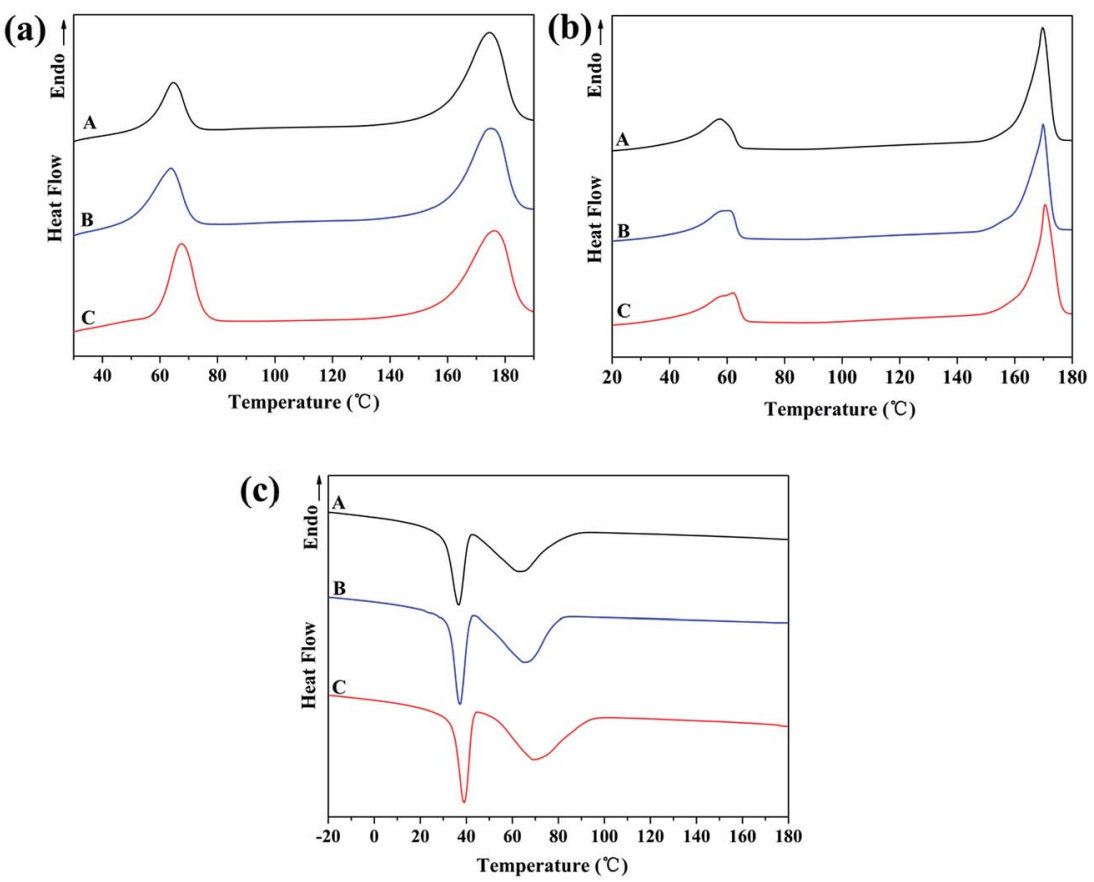

Fig. 3 DSC diagrams of PHBV/PEO electrospun fibers in different solvents: (A) CHL, (B) DCM, (C) DCM/EtOH. (a) The first heating cycle; (b) second heating cycle; (c) the cooling stage.

Table 3 Thermal properties of the PHBV/PEO electrospun fibers in different solvents $\left(T_{\mathrm{m} 1}=\right.$ the melting peak temperature of first heating scan; $T_{\mathrm{m} 2}=$ the melting peak temperature of second heating scan; $\chi=$ crystallinity calculated from first heating cycle; $T_{\mathrm{C}}=$ crystallization peak temperature from cooling scan)

\begin{tabular}{|c|c|c|c|c|c|c|c|c|c|c|}
\hline Sample & \multicolumn{6}{|c|}{ First heating } & \multicolumn{2}{|c|}{$\begin{array}{l}\text { First cooling } \\
T_{\mathrm{C}}\left({ }^{\circ} \mathrm{C}\right)\end{array}$} & \multicolumn{2}{|c|}{$\begin{array}{l}\text { Second heating } \\
T_{\mathrm{m} 2}\left({ }^{\circ} \mathrm{C}\right)\end{array}$} \\
\hline CHL & 64.6 & 174.6 & 17.0 & 50.6 & 22.9 & 66.3 & 36.7 & 63.2 & 59.6 & 169.7 \\
\hline DCM & 63.7 & 173.8 & 26.5 & 47.4 & 44.8 & 62.1 & 37.2 & 64.9 & 61.0 & 168.8 \\
\hline
\end{tabular}

than that in single solvent. The delay of the crystallization was associated to the complicated phase separation and fast drying times typical of the simultaneous electrospinning of two components in different solvents. ${ }^{36-38}$

\subsection{TGA analysis of PHBV/PEO electrospun fiber}

The TG and DTG curves of PHBV/PEO electrospun fiber in different solvents are listed in Fig. $4 \mathrm{a}$ and b, showing the degradation temperatures of PHBV and PEO, respectively.
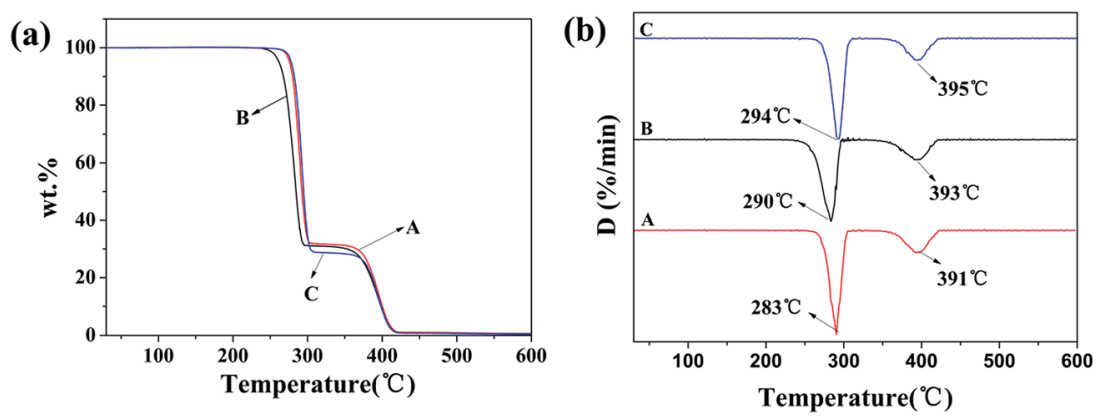

Fig. 4 TGA (a) and DTG (b) diagrams of PHBV/PEO electrospun fibers in different solvents: (A) CHL, (B) DCM, (C) DCM/EtOH. 
All the curves in Fig. 4 presented two distinctly distinguished thermal decomposition peaks ascribable to PHBV and PEO components. TGA and first derivatives of DTG for electrospun PHBV/PEO with different solvents showed that all samples exhibited a distinct weight loss stage of PHBV and PEO component at $267-307{ }^{\circ} \mathrm{C}$ and $350-415{ }^{\circ} \mathrm{C}$, the temperature of the maximum mass loss rate is $283-294{ }^{\circ} \mathrm{C}$ and $391-395{ }^{\circ} \mathrm{C}$, respectively. The TG and DTG values indicate that the $\mathrm{PHBV} /$ PEO electrospun fibers prepared with DCM/EtOH solvent showed higher degradation temperatures compared to the samples prepared with CHL and DCM alone, pointing to a better performance of binary-solvent system.

\subsection{Mechanical properties of PHBV/PEO electrospun fibers}

Mechanical properties of the PHBV/PEO electrospun fiber with different solution were shown in the Fig. 5. The results

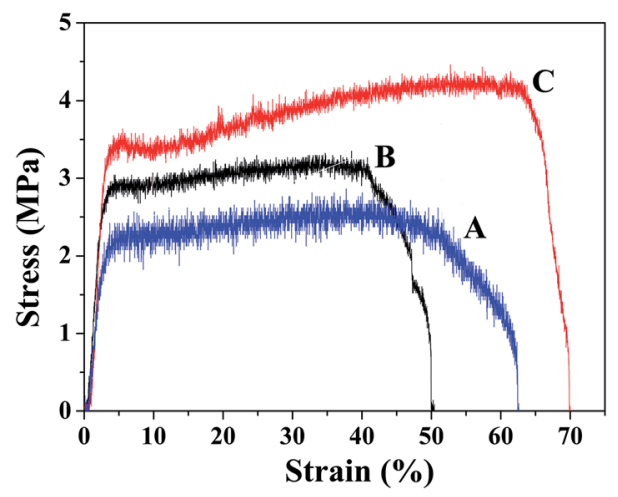

Fig. 5 Mechanical properties of PHBV/PEO electrospun fibers in different solvents: (A) CHL, (B) DCM, (C) DCM/EtOH. discovered that the PHBV/PEO electrospun fiber with the DCM/ EtOH solvent has higher tensile strength (4.46 MPa) and strain at break $(70.1 \%)$ compared with the fiber with the CHL (3.15 MPa, 62.6\%) and DCM (3.35 MPa, 50.4\%) solvent, the Young's modulus is basically identical with each other.

As reported, the mechanical properties of electrospun fibers are influenced by crystallinity and molecular orientation, and their crystal structure is controlled by the processing parameters and the electrical and rheological properties of the polymer solution. ${ }^{39}$ According to Arinstein and Zussman, ${ }^{40}$ the relaxation process that was generated by evaporation of solvent within electrospun fibers, can significantly affect the mechanical properties of polymeric nanofibers. From the Tables 2 and 3, the $\mathrm{PHBV} / \mathrm{PEO}$ electrospun fiber that are produced by using binarysolvent (DCM/EtOH) with lower evaporation rate, have smaller fiber diameter, higher degree of molecular orientation and crystallinity, and consequently the mechanical properties of electrospun fibers was superior to the fibers with single solvent (CHL, DCM). The similar results are discussed independently by Wong et al., ${ }^{41}$ which led to the conclusion that the degree of crystallinity and molecular orientation of fibers are enhanced when the diameter of spun fibers is reduced, resulting in improved mechanical strength.

\subsection{In vitro degradation of $\mathrm{PHBV} / \mathrm{PEO}$ electrospun fibers}

The degradation behavior of PHBV/PEO electrospun fibers with different solvents was investigated by incubating the samples in PBS at $37^{\circ} \mathrm{C}$. Fig. 6 and 7 have shown the morphology and weight loss of electrospun fibers after various time points during the in vitro degradation study, and the data for each sample was calculated by $T$-test or Student's $t$ test and $P<0.05$. The biodegradation
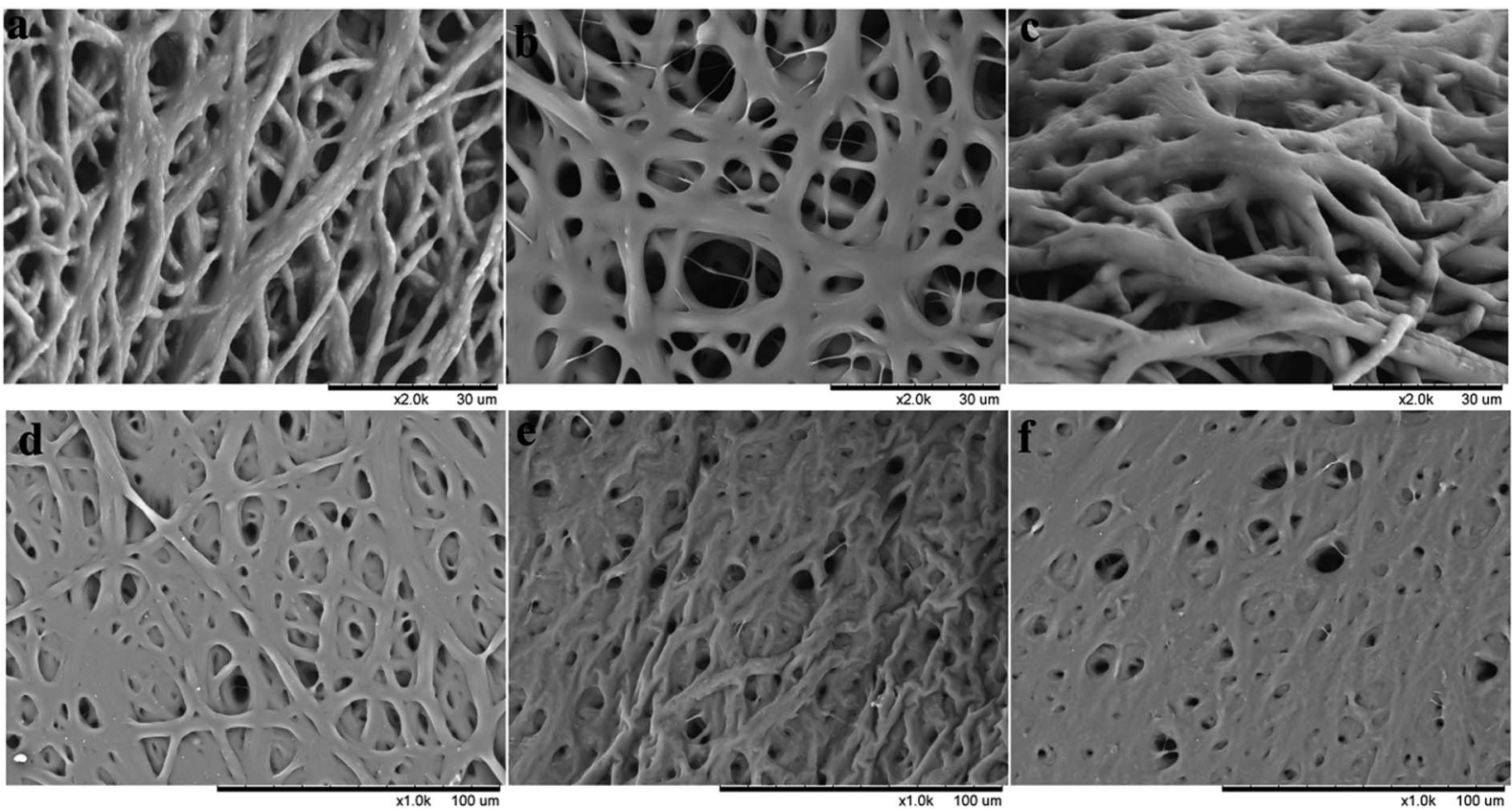

Fig. 6 SEM micrographs of PHBV/PEO electrospun fibers with different solvents after in vitro degradation: (a) 2 week, CHL ( $\times 1.0 k)$, (b) 2 week, $\operatorname{DCM}(\times 1.0 k)$, (c) 2 week, DCM/EtOH (x1.0k), (d). 4 week, CHL (×2.0k), (e) 4 week, DCM (×2.0k), (f) 4 week, DCM/EtOH ( $\times 2.0 k)$. 


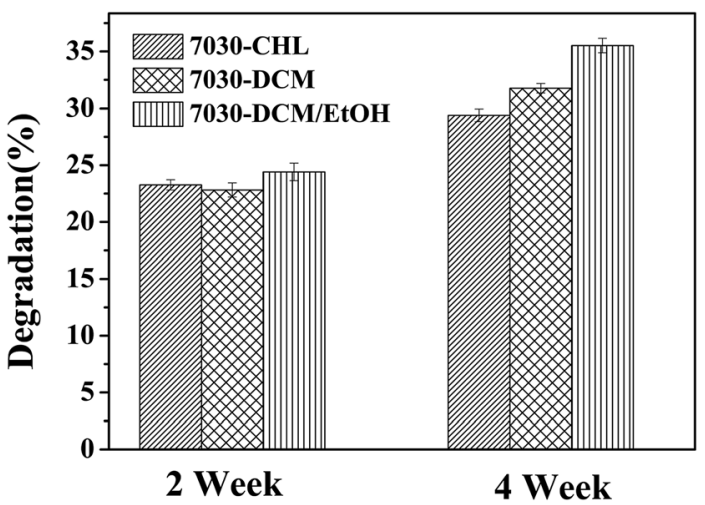

Fig. 7 The weight loss of PHBV/PEO electrospun fiber with different solvents after in vitro degradation $(P<0.05)$.

of polymers involves distinct mechanisms, depending on the nature of a polymer. For poly(hydroxyalkanoates) (PHA), hydrolysis (biotic or abiotic) followed by hydrodegradation is the primary pathway involved in the biodegradation. ${ }^{42}$
In Fig. 6a-c, all electrospun fibers appear to swell, but still keep the morphology of fibers. In the Fig. $6 \mathrm{~d}$ and e, the morphology of the PHBV/PEO electrospun fibers was gradually lost, but it can be observed the morphology of fibers partly after 4 weeks, while in the Fig. 6 f, the surface leveled off after 4 weeks, and there are some pores on the surface. Due to the excellent water-solubility and hydrophilic characters of PEO in the blend, that could help absorb and keep the water and preceded biodegradation of PHBV. It well agreed with the conclusion and mechanism reported by Li et al. ${ }^{43}$ and Ke et al. ${ }^{44} \mathrm{PEO}$, as the plasticizer, reduced the interaction between the chains of PHBV and PEO in the blends, thereby accelerating biodegradation. Another explanation could be the natural incompatibility between PHBV and PEO, already mentioned by Parra, ${ }^{42}$ and could account for the extensive biodegradation because of the migration of this plasticizer to the surface of the polymeric material. As exhibited in Fig. 7, the mass loss of PHBV/PEO electrospun fiber with CHL, DCM and DCM/EtOH solvent was $23.3 \%, 22.8 \%$ and 22.4 after 2 week degradation, and $29.4 \%$, $31.8 \%$ and $35.5 \%$ after 4 week degradation, respectively. This
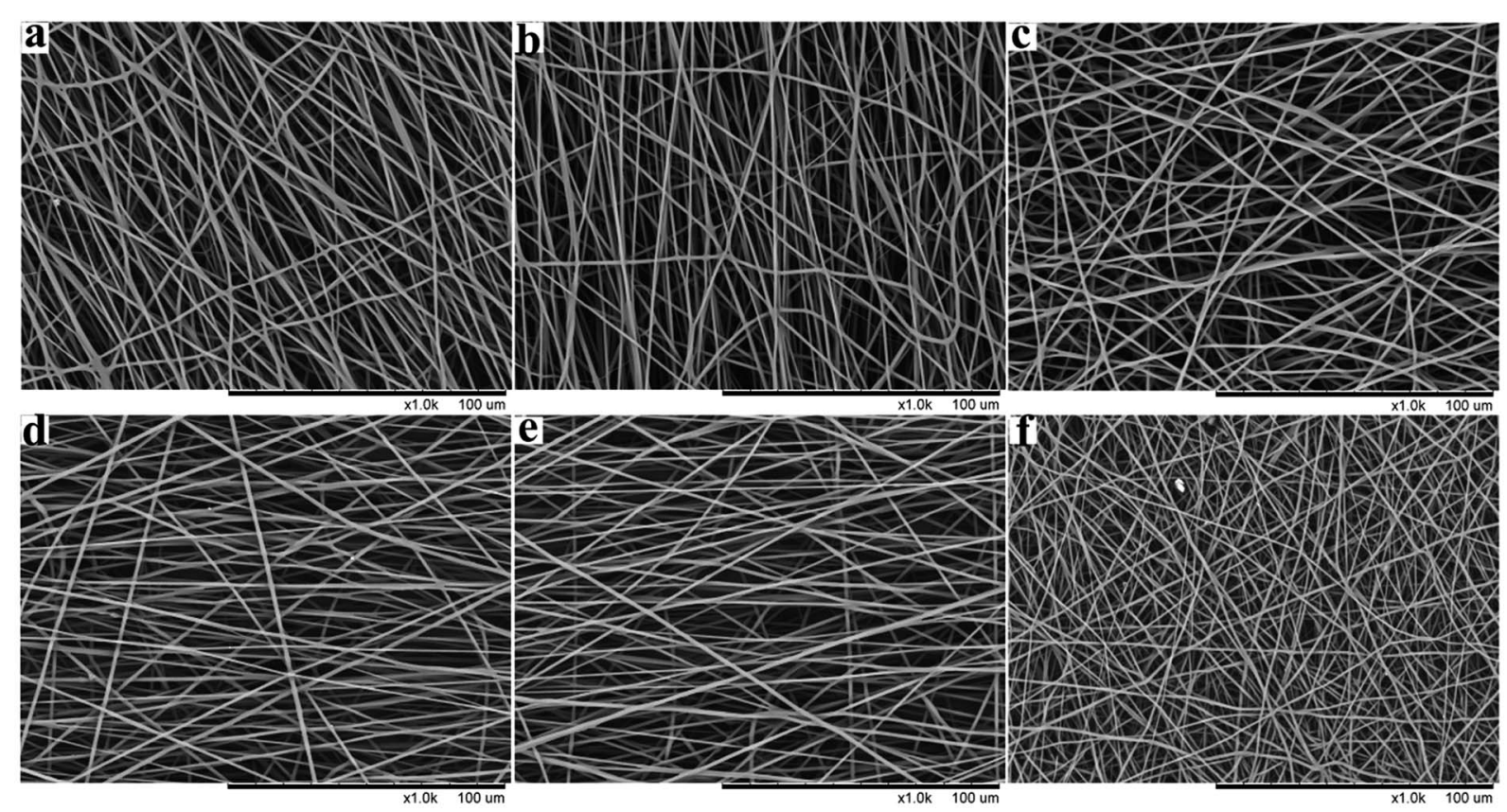

Fig. 8 Effect of solvent ratio (DCM/EtOH) on PHBV/PEO electrospun fiber morphology: (a) $9: 1$, (b) $8: 1$, (c) $7: 1$, (d) $6: 1$, (e) $5: 1$, (f) $4: 1$.

Table 4 Physical solution properties for 10\% w/v PHBV/PEO solution in different solvent ratios DCM/EtOH and mean fiber diameter

\begin{tabular}{|c|c|c|c|c|c|c|c|}
\hline $\begin{array}{l}\text { DCM/EtOH } \\
(\mathrm{v} / \mathrm{v})\end{array}$ & Solubility & $\begin{array}{l}\text { Electro- } \\
\text { spinnability }\end{array}$ & $\begin{array}{l}\text { Conductivity } \\
\left(\mu \mathrm{S} \mathrm{cm}^{-1}\right)\end{array}$ & $\begin{array}{l}\text { Viscosity } \\
\text { (mPa s) }\end{array}$ & $\begin{array}{l}\text { Surface tension } \\
\left(\mathrm{mN} \mathrm{m}^{-1}\right)\end{array}$ & $\begin{array}{l}\text { Vapor pressure } \\
\text { at } 20^{\circ} \mathrm{C}(\mathrm{mmHg})\end{array}$ & $\begin{array}{l}\text { Mean fiber } \\
\text { diameter (nm) }\end{array}$ \\
\hline $9: 1$ & + & + & $4.49 \pm 0.01$ & $103 \pm 2.5$ & $25.6 \pm 0.5$ & 342.9 & $813 \pm 169$ \\
\hline $8: 1$ & + & + & $4.59 \pm 0.01$ & $97 \pm 3.1$ & $25.4 \pm 0.5$ & 339.3 & $718 \pm 154$ \\
\hline $7: 1$ & + & + & $5.04 \pm 0.01$ & $82 \pm 3.5$ & $25.0 \pm 0.8$ & 334.7 & $660 \pm 153$ \\
\hline $6: 1$ & + & + & $5.11 \pm 0.01$ & $74 \pm 2.8$ & $24.8 \pm 0.3$ & 328.8 & $600 \pm 161$ \\
\hline $5: 1$ & + & + & $5.23 \pm 0.01$ & $67 \pm 3.8$ & $23.8 \pm 0.1$ & 320.9 & $560 \pm 171$ \\
\hline $4: 1$ & + & + & $5.45 \pm 0.01$ & $51 \pm 2.1$ & $22.5 \pm 0.1$ & 309.9 & $482 \pm 170$ \\
\hline $3: 1$ & - & - & $5.50 \pm 0.01$ & $45 \pm 2.5$ & $22.3 \pm 0.3$ & 293.4 & - \\
\hline
\end{tabular}


could be explained that DCM/EtOH, which has lower evaporation and higher conductivity than DCM and CHL, resulted in more stabilized electrospinning process. And thereby the distribution of PHBV and PEO fibers are more uniform than that with CHL and DCM solvent. Due to the hydrophilic of PEO, it was degraded in the first step of degradation, leading to more and uniform holes (surface defect) in the electrospun fibers when binary-solvent system (DCM/EtOH) was used as solvent,
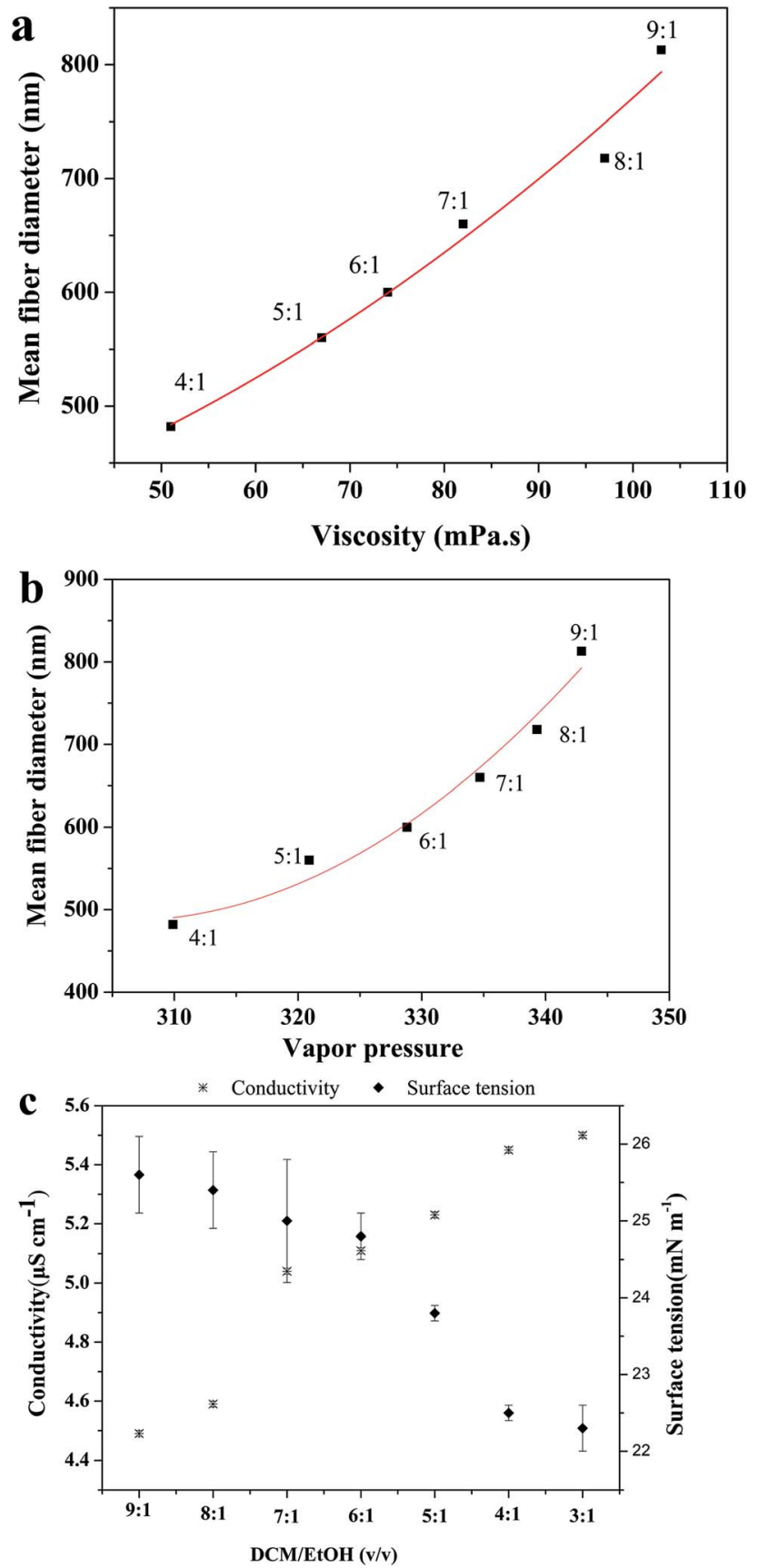

Fig. 9 (a) Effect of the viscosity of $\mathrm{DCM} / \mathrm{EtOH}$ in different ratios on PHBV/PEO mean fiber diameter; (b) effect of the vapor pressure of $\mathrm{DCM} / \mathrm{EtOH}$ in different ratios on PHBV/PEO mean fiber diameter; (c) effect of solvent ratio on the conductivity and surface tension of $\mathrm{PHBV} / \mathrm{PEO}$ solution. and then more PBS can fast penetrate to the spaces, and accelerate the degradation rate.

\subsection{The effect of solvent ratio (DCM/EtOH) on PHBV/PEO electrospun fiber}

From detailed studies of the PHBV/PEO electrospun fibers by using different solvents above, DCM/EtOH was found to produce defect-free fibers with the smallest mean diameter and a narrow diameter distribution. Therefore DCM/EtOH was mixed in a range of compositional ratios $(9: 1,8: 1,7: 1,6: 1$, $5: 1,4: 1$ and $3: 1 \mathrm{v} / \mathrm{v}$ ) and the effect on the PHBV/PEO electrospun fiber morphology was studied.

The SEM images of PHBV/PEO electrospun fibers from a rang of DCM/EtOH ratios are shown in Fig. 8, and the physical solution properties of PHBV/PEO solutions, dissolvable and mean fiber diameter are summarized in Table 4 . It was obviously found that the smooth defect-free fibers with narrow diameter distribution were collected by using different ratios of $\mathrm{DCM} / \mathrm{EtOH}$ solvent system in Fig. 8. But PHBV/PEO was insoluble in DCM/EtOH $3: 1$ solvent, the fiber can not be collected. $\mathrm{EtOH}$, as the second solvent in the mixed-solvent, has high boiling point and dielectric constant, and low surface tension (in the Table 1). Thus, as the amount of EtOH increasing in the DCM/EtOH system, the conductivity was increased, and the surface tension, viscosity, vapor pressure and mean fiber diameter was decreased in Table 4. The previous results showed that the fiber diameter of solvent system DCM/EtOH at a ratio of $6: 1 \mathrm{v} / \mathrm{v}$ was $783 \mathrm{~nm}$. A possible explanation for this discrepancy could be a slight difference in ambient conditions, such as temperature and humidity. ${ }^{45}$

As the literature reported, ${ }^{25,29,46,47}$ the polymer solutions which have the highest conductivity and lowest surface tension and viscosity yielded the uniform bead-free and thinnest fibers. Fig. 9 exhibited the effect of the solvent ratio on the solution conductivity, surface tension and viscosity. It was clear that the average fiber diameter decreased with decreasing viscosity, similar result has been reported by Zong et al. ${ }^{34}$ Due to the high boiling point and vapor pressure of EtOH, the DCM/EtOH in different ratios evaporated slowing from the ejected charged jet, leading to the viscoelastic properties of the jet to change and therefore the stretching of the jet to a much lower diameter. Similarly, the solution conductivity is one of the main parameters in the electrospinning process, the increase in the conductivity of solution results in production of bead-free uniform and thinner fibers. Since the viscous polymer solution is subjected to more stretching under the high electrical field due to the repulsion of the charges present on its surface, and more charges can be carried at higher solution conductivity. Conversely, the high surface tension resulted in the unstable of the jet, causing more beads appear on the electrospun mats. ${ }^{8,20,48,49}$

\section{Conclusions}

PHBV/PEO electrospun fibers were fabricated by using three solvents (CHL, DCM, DCM/EtOH). Of all solvent, smooth defectfree electrospun fibers were collected, while the thinnest fibers 
with narrow distribution were produced by binary-solvent (DCM/EtOH), which of the mean fiber diameter was $738 \mathrm{~nm}$. In addition, by using DCM/EtOH as solvent, the tensile stress and elongation break were $4.46 \mathrm{MPa}$ and $70.1 \%$; in vitro degradation was $35 \%$ after 4 week in PBS. In brief, the mechanical and degradation properties of PHBV/PEO electrospun fibers were improved by contrast with that with CHL and DCM solvent. Most importantly, the effect of different solvent ratios of DCM/EtOH on fiber morphology and diameter was also investigated. As the second solvent in the mixed-solvent, EtOH has high boiling point and dielectric constant, and low surface tension, resulting in the decrease of mean fiber diameter as the amount of EtOH increasing in the DCM/EtOH system. The finest mean fiber diameter was $483 \mathrm{~nm}$ when DCM/EtOH at a ratio of $4: 1 \mathrm{v} / \mathrm{v}$. It was clearly seen that the properties of solvent (such as conductivity, viscosity, surface tension and boiling point) played an important role in the electrospinning process. Therefore, DCM/EtOH was promising in serving as the solvent for electrospun PHBV/PEO, not only to reduce the toxicity of solvent, but also to produce more uniform and thinnest electrospun fibers, providing a fundamental criteria for tissue engineering.

\section{Acknowledgements}

The authors would like to acknowledge the research center for analysis \& measurement of Donghua University. This work is supported by National Key Research and Development Plan of China (2016YFB0303201).

\section{References}

1 N. Bhardwaj and S. C. Kundu, Electrospinning: A fascinating fiber fabrication technique, Biotechnol. Adv., 2010, 28, 325347.

2 W.-J. Li, J. A. Cooper Jr, R. L. Mauck and R. S. Tuan, Fabrication and characterization of six electrospun poly $(\alpha-$ hydroxy ester)-based fibrous scaffolds for tissue engineering applications, Acta Biomater., 2006, 2, 377-385.

3 Y. C. Ahn, S. K. Park, G. T. Kim, Y. J. Hwang, C. G. Lee, H. S. Shin, et al., Development of high efficiency nanofilters made of nanofibers, Curr. Appl. Phys., 2006, 6, 1030-1035.

4 K. Ramanathan, M. A. Bangar, M. Yun, W. Chen, N. V. Myung and A. Mulchandani, Bioaffinity sensing using biologically functionalized conducting-polymer nanowire, J. Am. Chem. Soc., 2005, 127, 496-497.

5 T. J. Sill and H. A. von Recum, Electrospinning: Applications in drug delivery and tissue engineering, Biomaterials, 2008, 29, 1989-2006.

6 A. Zonari, M. T. Cerqueira, S. Novikoff, A. M. Goes, A. P. Marques, V. M. Correlo, et al., Poly(hydroxybutyrateco-hydroxyvalerate) bilayer skin tissue engineering constructs with improved epidermal rearrangement, Macromol. Biosci., 2014, 14, 977-990.

7 Y. Ding, J. A. Roether, A. R. Boccaccini and D. W. Schubert, Fabrication of electrospun poly(3-hydroxybutyrate)/poly( $\varepsilon$ - caprolactone)/silica hybrid fibermats with and without calcium addition, Eur. Polym. J., 2014, 55, 222-234.

8 R. Casasola, N. L. Thomas, A. Trybala and S. Georgiadou, Electrospun poly lactic acid (PLA) fibres: Effect of different solvent systems on fibre morphology and diameter, Polymer, 2014, 55, 4728-4737.

9 C. J. Luo, M. Nangrejo and M. Edirisinghe, A novel method of selecting solvents for polymer electrospinning, Polymer, 2010, 51, 1654-1662.

10 S. Wang, P. Ma, R. Wang, S. Wang, Y. Zhang and Y. Zhang, Mechanical, thermal and degradation properties of poly(D,L-lactide)/poly(hydroxybutyrate-co-hydroxyvalerate)/ poly(ethylene glycol) blend, Polym. Degrad. Stab., 2008, 93, 1364-1369.

11 P. Kuppan, K. S. Vasanthan, D. Sundaramurthi, U. M. Krishnan and S. Sethuraman, Development of poly(3-hydroxybutyrate-co-3-hydroxyvalerate) fibers for skin tissue engineering: effects of topography, mechanical, and chemical stimuli, Biomacromolecules, 2011, 12, 3156-3165.

12 N. Nagiah, L. Madhavi, R. Anitha, C. Anandan, N. T. Srinivasan and U. T. Sivagnanam, Development and characterization of coaxially electrospun gelatin coated poly(3-hydroxybutyric acid) thin films as potential scaffolds for skin regeneration, Mater. Sci. Eng. C, 2013, 33, 4444-4452.

13 Y. Y. Shangguan, Y. W. Wang, Q. Wu and G. Q. Chen, The mechanical properties and in vitro biodegradation and biocompatibility of UV-treated poly(3-hydroxybutyrate-co-3hydroxyhexanoate), Biomaterials, 2006, 27, 2349-2357.

14 P. Kuppan, S. Sethuraman and U. M. Krishnan, Poly(3hydroxybutyrate-co-3-hydroxyvalerate)-based nanofibrous scaffolds to support functional esophageal epithelial cells towards engineering the esophagus, J. Biomater. Sci., Polym. Ed., 2014, 25, 574-593.

15 S. E. Moreira Catoni, K. N. Trindade, C. A. T. Gomes, A. P. T. Pezzin and V. Soldi, Influence of Poly(Ethylene Glycol) (PEG) on the Properties of Influence of Poly(3Hydroxybutyrate-co-3-Hydroxyvalerate) - PHBV, Polim.: Cienc. Tecnol., 2013, 23, 320-325.

16 W. Meng, S.-Y. Kim, J. Yuan, J. C. Kim, O. H. Kwon, N. Kawazoe, et al., Electrospun PHBV/collagen composite nanofibrous scaffolds for tissue engineering, J. Biomater. Sci., Polym. Ed., 2007, 18, 81-94.

17 K. Oh Hyeong, L. Ik Sang, K. Young-Gwang, M. Wan, J. Kyung-Hye, K. Inn-Kyu, et al., Electrospinning of microbial polyester for cell culture, Biomed. Mater., 2007, 2, S52.

18 K. Sombatmankhong, O. Suwantong, S. Waleetorncheepsawat and P. Supaphol, Electrospun fiber mats of poly(3-hydroxybutyrate), poly(3hydroxybutyrate-co-3-hydroxyvalerate), and their blends, $J$. Polym. Sci., Part B: Polym. Phys., 2006, 44, 2923-2933.

19 Y. Xu, L. Zou, H. Lu, Y. Wei, J. Hua and S. Chen, Preparation and characterization of electrospun PHBV/PEO mats: The role of solvent and PEO component, J. Mater. Sci., 2016, 51, 5695-5711.

20 A. S. Asran, M. Salama, C. Popescu and G. H. Michler, Solvent Influences the Morphology and Mechanical 
Properties of Electrospun Poly(L-lactic acid) Scaffold for Tissue Engineering Applications, Macromol. Symp., 2010, 294, 153-161.

21 S.-M. Jung, G. H. Yoon, H. C. Lee and H. S. Shin, Chitosan nanoparticle/PCL nanofiber composite for wound dressing and drug delivery, J. Biomater. Sci., Polym. Ed., 2015, 1-12.

22 M. Avella, E. Martuscelli and M. Raimo, Review Properties of blends and composites based on poly(3-hydroxy)butyrate (PHB) and poly(3-hydroxybutyrate-hydroxyvalerate) (PHBV) copolymers, J. Mater. Sci., 2000, 35, 523-545.

23 W. B. Liau and C. F. Chang, Casting solvent effect on crystallization behavior and morphology of poly(ethylene oxide)/poly(methyl methacrylate), J. Appl. Polym. Sci., 2000, 76, 1627-1636.

24 X. Zong, S. Ran, K.-S. Kim, D. Fang, B. S. Hsiao and B. Chu, Structure and Morphology Changes during in vitro Degradation of Electrospun Poly(glycolide-co-lactide) Nanofiber Membrane, Biomacromolecules, 2003, 4, 416-423.

25 J. M. Deitzel, J. Kleinmeyer, D. Harris and N. C. Beck Tan, The effect of processing variables on the morphology of electrospun nanofibers and textiles, Polymer, 2001, 42, 261-272.

26 X. Qin and D. Wu, Effect of different solvents on poly(caprolactone) (PCL) electrospun nonwoven membranes, J. Therm. Anal. Calorim., 2012, 107, 1007-1013.

27 M. M. Hohman, M. Shin, G. Rutledge and M. P. Brenner, Electrospinning and electrically forced jets. I. Stability theory, Phys. Fluids, 2001, 13, 2201-2220.

28 D. H. Reneker, W. Kataphinan, A. Theron, E. Zussman and A. L. Yarin, Nanofiber garlands of polycaprolactone by electrospinning, Polymer, 2002, 43, 6785-6794.

29 K. H. Lee, H. Y. Kim, Y. M. La, D. R. Lee and N. H. Sung, Influence of a mixing solvent with tetrahydrofuran and $N, N$-dimethylformamide on electrospun poly(vinyl chloride) nonwoven mats, J. Polym. Sci., Part B: Polym. Phys., 2002, 40, 2259-2268.

30 S. Megelski, J. S. Stephens, D. B. Chase and J. F. Rabolt, Micro- and Nanostructured Surface Morphology on Electrospun Polymer Fibers, Macromolecules, 2002, 35, 8456-8466.

31 T. Subbiah, G. S. Bhat, R. W. Tock, S. Parameswaran and S. S. Ramkumar, Electrospinning of nanofibers, J. Appl. Polym. Sci., 2005, 96, 557-569.

32 X. Geng, O.-H. Kwon and J. Jang, Electrospinning of chitosan dissolved in concentrated acetic acid solution, Biomaterials, 2005, 26, 5427-5432.

33 G. M. Kim, G. H. Michler, S. Henning, H. J. Radusch and A. Wutzler, Thermal and spectroscopic characterization of microbial poly(3-hydroxybutyrate) submicrometer fibers prepared by electrospinning, J. Appl. Polym. Sci., 2007, 103, 1860-1867.

34 X. Zong, K. Kim, D. Fang, S. Ran, B. S. Hsiao and B. Chu, Structure and process relationship of electrospun bioabsorbable nanofiber membranes, Polymer, 2002, 43, 4403-4412.

$35 \mathrm{H}$. Tsuji and Y. Ikada, Properties and morphologies of poly(Llactide): 1. Annealing condition effects on properties and morphologies of poly(L-lactide), Polymer, 1995, 36, 27092716.

36 C. Chen, L. Wang and Y. Huang, Morphology and thermal properties of electrospun fatty acids/polyethylene terephthalate composite fibers as novel form-stable phase change materials, Sol. Energy Mater. Sol. Cells, 2008, 92, 1382-1387.

37 J. G. Fernandes, D. M. Correia, G. Botelho, J. Padrão, F. Dourado, C. Ribeiro, et al., PHB-PEO electrospun fiber membranes containing chlorhexidine for drug delivery applications, Polym. Test., 2014, 34, 64-71.

38 A. Bianco, M. Calderone and I. Cacciotti, Electrospun PHBV/ PEO co-solution blends: Microstructure, thermal and mechanical properties, Mater. Sci. Eng. C, 2013, 33, 10671077.

39 G. H. Kim and H. Yoon, Effect of an auxiliary electrode on the crystalline morphology of electrospun nanofibers, Appl. Phys. Lett., 2008, 93, 023127.

40 A. Arinstein and E. Zussman, Electrospun polymer nanofibers: Mechanical and thermodynamic perspectives, J. Polym. Sci., Part B: Polym. Phys., 2011, 49, 691-707.

41 S.-C. Wong, A. Baji and S. Leng, Effect of fiber diameter on tensile properties of electrospun poly( $\varepsilon$-caprolactone), Polymer, 2008, 49, 4713-4722.

42 D. F. Parra, J. Fusaro, F. Gaboardi and D. S. Rosa, Influence of poly(ethylene glycol) on the thermal, mechanical, morphological, physical-chemical and biodegradation properties of poly(3-hydroxybutyrate), Polym. Degrad. Stab., 2006, 91, 1954-1959.

43 X. J. Loh, K. K. Tan, X. Li and J. Li, The in vitro hydrolysis of poly(ester urethane)s consisting of poly[(R)-3hydroxybutyrate] and poly(ethylene glycol), Biomaterials, 2006, 27, 1841-1850.

$44 \mathrm{Y}$. Ke, Z. Qu, G. Wu and Y. Wang, Thermal and in vitro degradation properties of the $\mathrm{NH}_{2}$-containing PHBV films, Polym. Degrad. Stab., 2014, 105, 59-67.

45 L. Van der Schueren, B. De Schoenmaker, Ö. I. Kalaoglu and K. De Clerck, An alternative solvent system for the steady state electrospinning of polycaprolactone, Eur. Polym. J., 2011, 47, 1256-1263.

46 T. Uyar and F. Besenbacher, Electrospinning of uniform polystyrene fibers: The effect of solvent conductivity, Polymer, 2008, 49, 5336-5343.

47 S. C. Byun, Y. J. Jeong, J. W. Park, S. D. Kim, H. Y. Ha and W. J. Kim, Effect of solvent and crystal size on the selectivity of ZSM-5/Nafion composite membranes fabricated by solution-casting method, Solid State Ionics, 2006, 177, 3233-3243.

48 Y. N. Jo and I. C. Um, Effects of solvent on the solution properties, structural characteristics and properties of silk sericin, Int. J. Biol. Macromol., 2015, 78, 287-295.

49 L. Lan-Xin, W. Yan-Yan, M. Xi, X. Zhong-Dang and H. NingPing, The effects of PHBV electrospun fibers with different diameters and orientations on growth behavior of bonemarrow-derived mesenchymal stem cells, Biomed. Mater., 2012, 7, 015002. 